Proceedings of the Royal Society of Edinburgh: Section $A$ is a general journal, and papers in all areas of mathematics will be considered. Papers to be considered for publication should be sent to the Publications Manager, The Royal Society of Edinburgh, 22 George Street, Edinburgh EH2 2PQ, Scotland.

A paper by more than one author must be submitted with a statement. signed by each author, to the effect that the paper in its entirety is approved by the joint authors and naming the author who will be responsible for correspondence with the Society.

Authors will receive fifty $(5())$ offprints free of charge, this number to be shared between joint authors. Additional offprints may be obtained, in units of fifty. at a fixed scale of prices given on a form which will be attached to the proof.

In view of the high cost of publication, authors must prepare their papers as concisely as possible. Manuscripts should be submitted in triplicate and preferably should be typewritten on one side of A4 paper. double spaced with adequate margins. Authors are advised to retain a copy of their papers as the Society cannot accept responsibility for any loss.

Every paper must be accompanied by a Synopsis. in general not exceeding two hundred words, which will be printed in small type at the beginning of the paper.

References within the text should be indicated by bold numbers in square brackets. e.g. [2] or [3. p. 167]. For style of references at end of text, see recent issues of Proceedings $A$.

Authors should ensure that punctuation carries through the mathematics in the proper manner. The use of hyphens should be consistent. In the text avoid such abbreviations as: iff. w.r.t., a.e.. $\forall . \exists$. and thm.

Footnotes should be avoided. Headings should not be underlined. Every effort should be made to avoid complicated subscripts, superscripts. ranges of summation and integration. Horizontal fraction signs should normally be avoided: use either solidus signs / or negative exponents. Replace $e^{t \ldots 1}$ by exp [...] if the expression in parenthesis is complicated. Simple formulae should not be displayed unless they require a formula number. Use the prime ' or $d / d x$, but preferably not a dot, to denote ordinary differentiation. If possible use subscripts to denote partial differentiation of $\partial / \partial x$ etc. Bars reaching over several letters should be avoided: use $\sqrt{ }()$ or the exponent $1 / 2$ for the square root. Sub-subscripts and super-superscripts should be avoided if possible: bars and other devices over indices cannot be supplied.

Note that confusion very often arises between $I$ (one) and $I$ (eli): 0 (zero) and $O$ (Capital oh): $\circ$ (composition) and $o$ (lower case oh): $x$ and $x: U$ and $U: c$ and $\subset: \epsilon$ (belongs to) and $\epsilon$ (epsilon): $\varnothing$ (empty set) and $\phi$ (phi):, and comma ,: prime' and ': $K$ and $\kappa: p$ and $\rho: w$ and $\omega: \sum$ (summation) and $\Sigma$ (capital sigma): $\Pi$ (product) and $\Pi$ (capital pi): $v$ (lower case vee) and $\nu$ (Greek $n u): a$ (lower case a) and $\alpha$ (Greek alpha): y (lower case y) and $\gamma$ (Greek gamma). Please provide pencilled indicators in the margin where necessary. Where capitals and lower case of the same shape have to be printed, please indicate accordingly. Show italics by single underlining (except in the formulae which are set up normally in italics), bold face/Clarendon by wavy underlining and Greek by red underlining.

The statement of theorems, lemmas, et cetera, will be printed in italics and should be underlined. In definitions key words only should be in italics.

Equations should be indicated by numbers in parentheses in the right-hand margin.

Proofs of papers will be sent to the author. The cost of authors corrections in excess of five per cent of the printers' charge for the setting of a particular paper will be charged to the author.

\title{
Copyright
}

(C) 1992 The Royal Society of Edinburgh and the authors of individual papers.

It is the policy of the Royal Society of Edinburgh not to charge any royalty for the production of a single copy of any one article made for private study or research. Specific permission will not be required for photocopying multiple copies of copyright material, to be used for bona fide educational purposes, provided this is done by a member of the staff of the university, school or other comparable institution, for distribution without profit to student members of that institution and provided the copies are made from the original journal. Requests for the copying or reprinting of any article for any other purpose should be sent to the Royal Society of Edinburgh, 22/24 George Street, Edinburgh EH2 $2 \mathrm{PQ}$ 


\section{PROCEEDINGS OF THE ROYAL SOCIETY OF EDINBURGH}

\section{(Section A)}

\section{CONTENTS}

M. FAIERMAN

A priori bounds for solutions of an elliptic equation

Peter Binev and Kurt JetTer

Cardinal interpolation with shifted three-directional box splines

205

SHEN YAOTIAN and YAN SHUSEN

Existence and boundedness of a minimiser for a constrained minimisa-

tion problem on $\mathbf{R}^{\mathrm{n}}$ with limiting exponent

XIAOdONG LiN, Joseph W.-H. So and Jianhong Wu

Centre manifolds for partial differential equations with delays

FERNANDO GIMÉNEZ

On the growth of the relative volume of a tube with its radius

GéraRd IoOsS and KLAUS KIRCHGÄSSNER

Water waves for small surface tension: an approach via normal form

YISONG YANG

The existence of a class of cosmic string solutions with infinite energy

Oscar P. BRuno and Fernando Reitich

Solution of a boundary value problem for the Helmholtz equation via

variation of the boundary into the complex domain

Manuel A. del Pino

A global estimate for the gradient in a singular elliptic boundary value problem

WiLliam C. Waterhouse

Differentiable functions on algebras and the equation $\operatorname{grad}(w)=$ $M \operatorname{grad}(v)$

Michael RenARDY

A centre manifold theorem for hyperbolic PDEs 